Meeting Report

\title{
International EMBO workshop on 'model organisms in cell death research', Obergurgl (Austria)
}

\author{
K Bianchi ${ }^{1}$, PJ Jost ${ }^{2}$, T Kaufmann ${ }^{\star, 3}$ and R Rutkowski ${ }^{4}$ \\ Cell Death and Differentiation (2009) 16, 1180-1183; doi:10.1038/cdd.2009.60
}

The EMBO Workshop, Model Organisms in Cell Death Research 31 January - 4 February 2009, Obergurgl, Austria.

The EMBO Workshop, 'Model Organisms in Cell Death Research' was held on 31 January-4 February 2009 in the village of Obergurgl, Austria. There was plenty of snow, and the conditions on piste were matched by the exciting talks and discussions indoors. Skiing performances were rather mixed - probably best exemplified by the fact that the 'Obergurgl Cup' team trophy ended up in Australia. However, there was no doubt about the high quality of science presented and discussed. This has been a truly outstanding meeting, covering cell death research in model organisms from yeast to mammals, and we are all indebted to Andreas Villunger who - with the help of Andreas Strasser and Gerry Melino - has done a magnificent job in setting up this workshop.

Guido Kroemer (Villejuif, France) opened the meeting. He briefly spoke about a theme that was to repeat during the meeting - that apoptotic proteins also have non-apoptotic roles; in particular, many apoptotic proteins play roles in stress responses. His main focus was the translocation of calreticulin (CRT) from the ER to the cell surface before other signs of apoptosis, and the fact that this contributes to the immunogenicity of tumour cells. The exposure of CRT on the cell surface is dependent on a number of steps, including the activation of PERK, the partial activation of caspase-8, the conformational activation of Bax and Bak, and SNAREdependent exocytosis of CRT. Disruption of any of these steps prevents CRT from being exposed at the cell surface and interferes with the immunogenicity of dying cells.

The focus of the first session was cell death and evolution, with discussions mainly on model organisms that are not so established in cell death research. In Hydra, cells that fail to form buds owing to lack of nutrients die of apoptosis. Angelika Boettger (Munich, Germany) discussed efforts to identify apoptotic proteins in the recently sequenced genome of this multicellular organism. To date, 10 caspases, most of which look like caspase-3 homologues, have been identified. Interestingly, none contain a CARD domain. Members of the $\mathrm{Bcl}-2$ family have also been identified, two of which, $\mathrm{HyBcl}-2$ and HyBax, seem to localise to outer mitochondria, implying a role for mitochondria in Hydra apoptosis.

Steve Hand (Baton Rouge, USA) addressed the question of how do Artemia franciscana embryos survive extended periods of anoxia without inducing apoptosis. He presented evidence that these embryos have multiple mechanisms in place that prevent apoptosis from occurring. For example, mitochondria from $A$. franciscana embryos do not permeabilise and release cytochrome $c$ in response to high levels of calcium, cytochrome c does not activate caspase activity, and high levels of GTP inhibit caspase 9 activity.

To study apoptotic and non-apoptotic roles for caspases during Xenopus development, Mathias Fransen (Ghent, Belgium) introduced a GFP-based, non-invasive in vivo reporter system for caspase activation in embryonic development. Not all GFP-positive cells die and some appear to be circulating red blood cells, suggesting that caspases also play roles in differentiation.

It is clear that in mammals, the mitochondrial pathway is a major mechanism for apoptosis induction. However, it seems that mitochondria do not play such a key role in apoptosis in either Drosophila or in Caenorhabditis elegans. In the last talk of this session, Doug Green (Memphis, USA) discussed ongoing efforts to understand when did the mitochondrial pathway evolve.

Identifying new components and pathways of apoptosis in C. elegans was the focus of the second session. Barbara Conradt (Hanover, USA) began by presenting work functionally linking asymmetric cell division with cell death of the NSM sister cell. A new regulator of NSM sister cell death, dnj-11 (a MIDA1 homologue), has been identified and shown to cooperate with ces-2 to inhibit ces-1-dependent transcription of egl-1 in the NSM cell. As the NSM sister cell lacks ces-2 and $d n j-11, e g l-1$ is transcribed and death results. The NSM sister cell is half the size of the NSM cell owing to an asymmetric cell division, and interestingly, $d n j-11$, ces-1, and ces-2 are also required for this process.

\footnotetext{
${ }^{1}$ The Breakthrough Toby Robins Breast Cancer Research Centre, Institute of Cancer Research, London, UK; ${ }^{2}$ Molecular Genetics of Cancer Division, The Walter and Eliza Hall Institute of Medical Research, Parkville, Victoria, Australia; ${ }^{3}$ Institute of Pharmacology, Medical Faculty, University of Bern, Bern, Switzerland; ${ }^{4}$ Wellcome Trust Centre for Gene Regulation and Expression, College of Life Sciences, University of Dundee, Dundee, UK

${ }^{*}$ Corresponding author: T Kaufmann, Institute of Pharmacology, University of Bern, Friedbühlstrasse 49, CH-3010, Bern, Switzerland. Tel: + 41316323289 ;

Fax: +4131632 49 92; E-mail: thomas.kaufmann@pki.unibe.ch
} 
The remaining talks in this session discussed pathways involved in DNA-damage-induced apoptosis in the C. elegans germline. Brent Derry (Toronto, Canada), Rachael Rutkowski (Dundee, UK), Anton Gartner (Dundee, UK), and Michael Hengartner (Zürich, Switzerland) all discussed the ongoing efforts at identifying novel regulators of DNA-damage-induced apoptosis. Brent Derry and Anton Gartner each spoke of alternative, non-CEP-1/p53-dependent pathways that are required for apoptosis after DNA damage. Together, these studies imply that, in addition to the CEP-1/p53 induction of egl-1, there are other parallel pathways that are also required for an efficient apoptotic response after DNA damage. Rachael Rutkowski discussed the findings that further show that germline developmental signals can regulate CEP-1/p53induced apoptosis. Michael Hengartner wrapped up this session with a discussion on how hypoxia is linked to CEP1/p53-dependent apoptosis and with the interesting finding that sensory neurons can somehow signal to the germline to regulate apoptosis.

The third session was dedicated to Drosophila as a model system in cell death. Andreas Bergmann (Houston, USA) opened the session with a study on cell death induced by the IAP-antagonist, Hid, which when overexpressed in the developing eye results in a collapsed eye because of ectopic apoptosis. Carrying out a genetic screen to identify genes that are required for Hid-mediated killing, the Bergmann lab identified lid (little imaginal discs). In lid mutants, Hid killing is suppressed and these animals also display diminished cell death during development. It was found that lid mutants harbour lower levels of the initiator caspase, Dronc. Their data are consistent with a model, in which Hid kills by neutralising DIAP1 while triggering Lid-mediated induction of Dronc gene expression. This ultimately shifts the balance between DIAP1:Dronc in favour of Dronc, leading to Dronc-mediated cell death.

Cells not only die by apoptosis, but can also be eliminated by cell death that involves autophagy, a topic that was introduced by Eric Baehrecke (Worcester, USA). In a screen to uncover genes required for autophagy-mediated involution of salivary glands (SG), he identified ddlc1. The ddlc1-mutant animals displayed significantly delayed SG involution. The ddlc1 encodes a dynein light chain subunit, which seems to be essential for the formation of the autophagosome. As a consequence, ddlc1 mutants contain fibrillar aggregates, revealing a problem in protein clearance.

Using Dronc as bait in a large-scale affinity assay coupled to mass spectrometric analysis, Mariam Orme (London, UK) identified Crinkled (Ck) as a potential Dronc regulatory protein. Genetic analysis shows that $c k$ is indeed required for cell death induced by Hid-mediated apoptosis as well as cell death induced by RNAi-mediated depletion of DIAP1. Clearly, DIAP1 is the key cell death suppressor in Drosophila. Although DIAP1 interacts efficiently with caspases, it remains elusive how it mediates their inhibition.

Pascal Meier (London, UK) reported that DIAP1-mediated conjugation of ubiquitin (Ub) to effector caspases results in the suppression of their activity, most likely through stearic occlusion of the substrate-binding pocket and Ub-mediated conformational changes. Surprisingly, ubiquitylated caspases are not degraded by the proteasome, which leads to the possibility that de-ubiquitylating proteases can reactivate caspases after death stimuli.

The role of p53 family members in the control of cell death was the topic of the next session. It was opened by Domenico Migliorini (Ghent, Belgium), who presented in vivo data on the function of COP1. This protein was previously reported to cause an Mdm2-independent ubiquitylation of p53, thereby targeting this tumour suppressor for proteasomal degradation.

The next talk by Eleonora Candi (Rome, Italy) focused on the ability of micro RNA 203 (miR-203) to regulate $\mathrm{p} 63$ protein levels during skin development. The miR-203 specifically targets human and mouse p63 $3^{\prime}$-UTRs and is thereby able to regulate the proliferative capacity of human epidermal cells. Furthermore, Candi presented data on the WW domaincontaining E3 Ub ligase 1 (WWP1), which binds and targets p63 for Ub-mediated proteasomal degradation while accumulating in the nucleus after DNA-damage.

Andreas Villunger (Innsbruck, Austria) presented data from the first analysis of gene-targeted mice deficient for the p53induced protein with a death domain (PIDD). PIDD has been implicated as an activator of Caspase-2 in apoptosis induction, in response to DNA double strand breaks. However, Villunger and colleagues could not detect an essential role for PIDD in $\gamma$-radiation-induced thymocyte apoptosis. He pointed out recent evidence that PIDD and caspase- 2 could instead play a critical role in the DNA-damage-induced G2/M cell cycle arrest.

The final talk of the session was given by Tom Look (Boston, USA), who presented data from a p53-sensitised in vivo genetic screen in zebrafish that was employed to identify novel regulators of DNA-damage-induced apoptosis. Checkpoint kinase 1 (CHK1) was identified as a protein regulating a DNA-damage-activated nuclear apoptotic signalling pathway that is independent of p53 and known regulators of the mitochondrial cell death pathway. Knockdown or pharmacological inhibition of CHK1 was sufficient to restore $\gamma$-radiation-induced apoptosis in a p53-mutant zebrafish model. Responsiveness of the CHK1-regulated pathway in human tumour cells to pharmacological inhibition indicates its potential as a drug target.

The first of two sessions on Oncogenes and Tumour Suppressors was opened by Karen Vousden (Glasgow, UK), who reported on the contribution of p53 to the survival of stressed cells. Under conditions of low or transient stress, p53 can induce the expression of TIGAR, a protein with antioxidant activities. The TIGAR expression shifts glucose metabolism towards the pentose phosphate pathway, resulting in a decrease of intracellular ROS levels. TIGAR thus protects cells from ROS-induced apoptosis. Interestingly, some tumour cell lines overexpress TIGAR in a p53independent way, suggesting that TIGAR may contribute to tumour development, and so might present a potential target for cancer therapy.

Gerry Melino (Rome, Italy and Leicester, UK) presented data on the TAp73-selective knockout mouse. Unlike mice lacking all p73 isoforms, TAp73-/- mice have an increased lifespan and show a high incidence of spontaneous tumours, clearly identifying TAp73 as a tumour suppressor. Moreover, TAp $73^{-/-}$mice are infertile and produce oocytes exhibiting 
spindle abnormalities. The molecular mechanism involves physical interaction with kinetochore proteins (BubR1, Bub1, and Bub3), affecting the Spindle Assembly Checkpoint, causing aneuploidy.

Using a p53ER ${ }^{\text {TAM }}$ knockin mouse model, wherein the p53 expression can be induced, Gerard Evan (San Francisco, USA) showed the presence of persistent p53-activating signals in tumours and the need for a tumour to constantly shut off p53 levels. He then presented an elegant study on a mouse model expressing Myc constitutively under the weak Rosa26 promoter. He showed that very low levels of deregulated Myc expression are much more oncogenic than overexpressed Myc. It seems that low levels of Myc fail to activate the ARF-mediated tumour suppressor pathway and induce oncogenesis without increasing apoptosis. In another mouse model that mimics anti-Myc therapy in the mouse ('omo Myc' mouse, in which the Myc expression can be switched off), inactivation of Myc lead to dramatic, but well tolerated, side effects in certain cells or tissues (for example, sperm, skin), but had no negative impact on the general health or total weight and, importantly, did not alter blood chemistry. Impressively, these side effects were rapidly and completely reversed upon restoration of Myc function. Shutting off Myc in a KRas-induced lung tumour model lead to rapid regression of tumours in a p53-independent manner. However, only about $35 \%$ of tumours were eradicated by Myc inhibition. Nonetheless, those tumours that did grow back remained completely susceptible to second and, indeed, a third round of Myc inhibition.

The second session on Oncogenes and Tumour Suppressors was mainly dedicated to the role of $\mathrm{Bcl}-2$ family members in tumour development and sustained tumour growth. Andreas Strasser (Melbourne, Australia) pointed out how different endogenously expressed $\mathrm{Bcl}-2$ proteins can

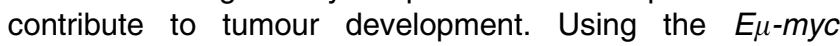
transgenic mouse model of lymphomagenesis, he illustrated well the fact that deletion of $\mathrm{Bcl}-\mathrm{x}_{\mathrm{L}}$ in the haematopoietic system is able to completely abrogate tumour formation in this model. In contrast, deletion of Bcl-2 had no impact on lymphomagenesis and survival of experimental animals, indicating the non-redundant roles of individual pro-survival $\mathrm{Bcl}-2$ family members. Interestingly, pharmacological blockade of Bcl- $x_{L}$ with the BH3-mimetic drug, ABT-737, was able to protect $E \mu$-myc transgenic mice from tumour formation.

Anna Frenzel (Innsbruck, Austria) investigated the roles of the $\mathrm{BH}$ 3-only proteins, Bmf and Bad, in tumour formation in the $E \mu-m y c$ transgenic mouse model. Although $\mathrm{Bmf}^{-1}$ mice have abnormally elevated levels of $B$ cells, they do not develop any malignancy; however, loss of Bmf markedly accelerated lymphoma development in E $\mu$-myc transgenic mice. Interestingly, although $\mathrm{Bad}^{-/}$mice have a normal lymphoid compartment, loss of Bad also accelerated tumour development in this transgenic mouse model.

Verena Labi (Innsbruck, Austria) showed that, surprisingly, the deletion of another BH3-only protein, PUMA, completely abrogated $\gamma$-radiation-induced thymoma development. In contrast, loss of p53 greatly accelerated tumour development in this setting. Verena Labi reports that PUMA deletion protects most haematopoietic cell types, including thymocytes, from $\gamma$-radiation-induced apoptosis and thereby greatly limits the proliferative response of haematopoietic stem and progenitor cells. The reduction of premalignant progenitor cell proliferation may translate into a reduced thymoma incidence in $P U M A^{-1-}$ mice.

Alexander Egle (Salzburg, Austria) illustrated the function of protein kinase $\mathrm{C}$ (PKC) $\beta$ as an important player in chronic lymphoid leukaemia (CLL) and its potential as a target for treatment of CLL patients. Transgenic mice overexpressing TCL1 develop B-CLL. Remarkably, Egle and colleagues showed that loss of PKC $\beta$ greatly inhibited TCL1-induced lymphoma development. The killing of human CLL cells in vitro by a $\operatorname{PKC} \beta$ inhibitor underscored this finding, pointing towards $\mathrm{PKC} \beta$ as a potential drug target in CLL.

The final talk of the session was presented by Martin Schuler (Essen, Germany), who focused on pharmacological inhibition of Bcl-2 family members in Rituximab-resistant $\mathrm{B}-\mathrm{NHL}$ cells using human B-NHL cell lines in vitro and in a xenotransplant model. By treatment with the $\mathrm{BH} 3$ mimetic, ABT-737, Rituximab-resistant cell lines were resensitised, whereas others responded to PI3K inhibitors. This implies that resistance patterns can be overcome by pharmacological inhibition of $\mathrm{Bcl}-2$ prosurvival family members or their upstream regulators.

Thomas Decker (Vienna, Austria) launched the session on Cell Death \& Immunity, reporting that mice defective in type I interferon response are protected from lethal infection with Listeria monocytogenes. A new role for type I interferons was proposed in increasing a non-apoptotic (necrotic-like) form of cell death in infected macrophages through production of nitric oxide and sensitisation of macrophages to the pore-forming activity of the pathogen's haemolysin, Listeriolysin $O$.

Georg Häcker (Münich, Germany) presented an elegant model to study apoptosis in murine neutrophils differentiated in vitro from Hoxb8-immortalised myeloid progenitors. Generating neutrophils ex vivo from genetically modified mice, the BH3-only proteins, Bim and Puma, were identified as the main proapoptotic initiators of spontaneous neutrophil apoptosis. Using a pneumococcal meningitis model in the mouse, it was shown that neutrophils overexpressing Bcl-2 maintain inflammation within the CNS, leading to stronger brain damage by preventing uptake of neutrophils by macrophages.

Jose Yelamos (Barcelona, Spain) showed that mice deficient for PARP2 (but not for PARP1) contain less $\mathrm{CD}^{+}{ }^{+} \mathrm{CD}^{+}$double-positive thymocytes, associated with an increased sensitivity of DP thyomcytes to apoptotic stimuli. Microarray analysis revealed a transcriptional induction of the proapoptotic BH3-only proteins, Noxa and Puma.

The session was concluded by the talk of Eric Eldering (Amsterdam, The Netherlands), who proposed an important role for the BH3-only protein, Noxa, in the death or survival decision of expanding T-cell clones. TCR-stimulation of murine $T$ cells lead to a p53-independent induction of Noxa. $\mathrm{Noxa}^{-1-}$ mice accumulated effector $\mathrm{T}$ cells over time, and antigen challenge led to the persistence of otherwise eliminated subdominant clones. It was proposed that Noxa is important in this antigen-driven interclonal T-cell competition, thereby ensuring an effective immune response.

In mammals, the decision between life and death relies on the balance between pro- and anti-apoptotic members of the Bcl-2 protein family. David Huang (Melbourne, Australia) 
opened the first session on Molecular Mechanisms of Cell Death and discussed the structure of the $\mathrm{BH} 3$ domain. $\mathrm{He}$ presented new findings using the pharmacological $\mathrm{BH} 3$ mimetic, ABT-737, in a mouse lymphoma model. Testing in a mouse lymphoma model showed that this compound has potent anti-tumour activity in combination with low doses of chemotherapeutic drugs. Interestingly, certain viruses also express proteins with structural similarities to anti-apoptotic Bcl-2 family members, and are thus able to bind and neutralise proapoptotic family members. For example, BHRF-1, encoded by EBV, can block apoptosis by binding to Bak and Bim. Importantly, these virally encoded proteins may well form potential targets for the treatment of lymphomas or other virally induced tumours.

Jochen Prehn (Dublin, Ireland) proposed a model, on the basis of imaging and mathematical modelling approaches, in which a minimal Bax (or Bak) activation may be sufficient to trigger irreversible apoptotic responses. He showed by FRET and quantitative confocal microscopy analysis that Bax translocation and oligomerisation starts just before or at about the same time as MOMP, and that most of the Bax translocation and oligomerisation actually occurs downstream of MOMP. He further presented data that, although poreformation kinetics being rapid, MOMP often proceeds in a wave-like fashion through the cell.

Christoph Borner (Freiburg, Germany) challenged the model, in which apoptosis is induced by lysosomal membrane permeabilisation (LMP) and subsequent release of lysosomal cathepsins into the cytosol. He showed that LMP is a consequence of apoptosis rather than an initiating event. Bax/Bak double knockout cells do not seem to undergo LMP in response to IL-3 withdrawal, or exposure to UV and etoposide. His data suggest that LMP requires MOMP. Hence, LMP and release of cathepsin seem to enhance, but not initiate, apoptosis.

Using an RNAi screen targeting the human kinome, Miguel Martins (Leicester, UK) identified the Ste20-family kinase, MAP4K3, as a pro-apoptotic kinase that plays a role in DNA-damage-induced cell death. By the regulation of mTORC1, MAP4K3 seems to stimulate post-transcriptional upregulation of Bad and Puma. Moreover, MAP4K3 also triggers JNK-dependent and phospho-mediated activation of Bim. Interestingly, MAP4K3 is mutated, downregulated, in pancreatic cancers, likely contributing to their resistance to apoptosis.

Patrice Petit (Paris, France) presented data indicating that mature cardiolipins (CLs) are required for the formation and the full functional activity of a newly discovered cell death activating platform at the mitochondrial contact sites involved in Fas-mediated apoptosis. The data arise from a study of Barth syndrome patient-derived lymphoblasts and HeLa cells (wherein the TAZZ gene has been deleted) that have nonmature CL and present a complete inhibition of apoptosis. To support this idea, Patrice Petit developed a new model system by generating Giant Unilamellar Vesicles, which led to the identification of $C L$ as an essential lipid in providing a platform for the association of caspase-8 and Bid-FL on the outer mitochondrial membrane during cell death.
The E3 ligases, clAP1 and TRAF2, are required for TNF $\alpha$-mediated activation of NF- $\kappa \mathrm{B}$. To study the individual contribution of clAP1 and TRAF2 in Ub-mediated activation of $\mathrm{NF}-\kappa \mathrm{B}$, James Vince and John Silke (Melbourne, Australia) identified a motif within TRAF2 required for clAP interaction. TRAF2 $^{-1}$ MEFs reconstituted with either a TRAF2 RING mutant (TRAF2 ${ }^{\text {RINGmut }}$ ) or TRAF2 clAP-binding mutant were then tested for their ability to rescue $T_{R A F 2}{ }^{-}$cells. Remarkably, TRAF2 $2^{\text {INGmut }}$ was able to restore TNF $\alpha$ mediated NF- $\kappa$ B activation, but a clAP-binding mutant was not, indicating that TRAF2's E3-ligase activity was not required for TNF $\alpha$-mediated activation of NF- $\kappa \mathrm{B}$, but clAP's E3 ligase activity was. Despite the fact that a TRAF2 RINGmut rescued the activation of NF- $\kappa \mathrm{B}$ in response to TNF, it was unable to protect the cells from the lethal effect of TNF $\alpha$, showing (for the first time) that protection from TNF can be separated from activation of NF- $\kappa \mathrm{B}$.

Tae-Bong Kang (Rehovot, Israel) showed that caspase-8 also plays important non-apoptotic roles. In particular, caspase- 8 is required for macrophage differentiation, liver regeneration after partial hepatectomy, withholding transformation of MEFs in culture, and growth enhancement of $B$ lymphocytes in response to LPS. Moreover, a mutant of caspase- 8 that cannot be cleaved between the p20 and p10 subunits fails to induce cell death, but is just as capable as wild-type caspase-8 in assisting LPS-induced B-cell growth response as well as various other non-apoptotic functions of this enzyme.

Philipp Jost (Melbourne, Australia), Thomas Kaufmann (Bern, Switzerland), and Nadia Corazza (Bern, Switzerland) all covered cell death of hepatocytes. In this system, Fasinduced killing requires caspase-8-mediated Bid cleavage, which results in tBid-induced MOMP and cell death. In the absence of Bid, hepatocytes are resistant to Fas-induced cell death. Philipp Jost has found that XIAP is responsible for the resistance of $\mathrm{Bid}^{-1-}$ hepatocytes to Fas treatment. In the absence of XIAP, Bid $^{-1-}$ cells are resensitised to Fas-induced cell death. Thomas Kaufmann identified Bim as an additional determinant for apoptosis induced by TNF $\alpha$, using the LPS plus $\mathrm{D}(+)$-galactosamine (GaIN) model in the mouse. Genetic deletion of Bid was insufficient to protect hepatocytes from cell death induced by the combined treatment of LPS and GalN. On the other hand, mice lacking both Bid and Bim were significantly protected. In agreement with the important role played by Bim, Nadia Corazza found that Bim is required for TRAIL-induced cell death after exposure to high doses of paracetamol. The next talk in the session also focussed on this model, but from a different point of view. Rui Castro (Lisbon, Portugal) identified miRNA21 as an important factor in liver regeneration. In addition, miRNA21 expression is modulated by bile acids, known to be important modulators of life/death decisions in hepatocytes.

Acknowledgements. We like to thank the authors for giving us permission to include unpublished work. K.B. is supported by a FEBS long-term post-doctoral fellowship, P.J.J. is supported by the Mildred-Scheel Stiftung/Deutsche Krebshilfe, T.K. is supported by the Swiss National Science Foundation (PP0033_119203), and R.R. is supported by CRUK. 